\title{
METALLICITY GRADIENT OF A LENSED FACE-ON SPIRAL GALAXY AT REDSHIFT 1.49
}

\author{
T.-T. Yuan ${ }^{1}$, L. J. Kewley ${ }^{1}$, A. M. Swinbank ${ }^{2}$, J. Richard ${ }^{2,3}$, AND R. C. Livermore ${ }^{2}$ \\ ${ }^{1}$ Institute for Astronomy, University of Hawaii, 2680 Woodlawn Drive, Honolulu, HI 96822, USA \\ 2 Institute for Computational Cosmology, Department of Physics, Durham University, South Road, Durham DH1 3LE, UK \\ ${ }^{3}$ Dark Cosmology Centre, Niels Bohr Institute, University of Copenhagen, Juliane Maries Vej 30, 2100 Copenhagen, Denmark \\ Received 2011 January 26; accepted 2011 March 14; published 2011 April 11
}

\begin{abstract}
We present the first metallicity gradient measurement for a grand-design face-on spiral galaxy at $z \sim 1.5$. This galaxy has been magnified by a factor of $22 \times$ by a massive, X-ray luminous galaxy cluster MACS J1149.5+2223 at $z=0.544$. Using the Laser Guide Star Adaptive Optics aided integral field spectrograph OSIRIS on KECK II, we target the $\mathrm{H} \alpha$ emission and achieve a spatial resolution of $0^{\prime \prime} 1$, corresponding to a source-plane resolution of $170 \mathrm{pc}$. The galaxy has well-developed spiral arms and the nebular emission line dynamics clearly indicate a rotationally supported disk with $V_{\text {rot }} / \sigma \sim 4$. The best-fit disk velocity field model yields a maximum rotation of $V_{\text {rot }} \sin i=$ $150 \pm 15 \mathrm{~km} \mathrm{~s}^{-1}$, and a dynamical mass of $M_{\mathrm{dyn}}=(1.3 \pm 0.2) \times 10^{10} \operatorname{cosec}^{2}(i) M_{\odot}$ (within $2.5 \mathrm{kpc}$ ), where the inclination angle $i=45^{\circ} \pm 10^{\circ}$. Based on the $[\mathrm{N} \mathrm{II}]$ and $\mathrm{H} \alpha$ ratios, we measured the radial chemical abundance gradient from the inner hundreds of parsecs out to $\sim 5 \mathrm{kpc}$. The slope of the gradient is $-0.16 \pm 0.02 \mathrm{dex} \mathrm{kpc}^{-1}$, significantly steeper than the gradient of late-type or early-type galaxies in the local universe. If representative of disk galaxies at $z \sim 1.5$, our results support an "inside-out" disk formation scenario in which early infall/collapse in the galaxy center builds a chemically enriched nucleus, followed by slow enrichment of the disk over the next 9 Gyr.
\end{abstract}

Key words: galaxies: abundances - galaxies: evolution - galaxies: high-redshift - gravitational lensing: strong

Online-only material: color figures

\section{INTRODUCTION}

The spatial distribution of heavy element abundances is a unique tool to trace the structure formation history of disk galaxies. Since the very early work of Searle (1971), Shields (1974), and Zaritsky et al. (1994), it has been well established that there is a negative radial metallicity gradient in spiral galaxies such that the central disk region is more metal-enriched than the outer regions. The evolution of this gradient over cosmic time provides a powerful constraint on disk formation and evolution models.

Sophisticated chemical evolution models (CEMs) have been successful in reproducing the current gradient of local galaxies, but different models disagree on the time variation of the radial metallicity gradient (e.g., Molla et al. 1996; Chiappini et al. 1997; Magrini et al. 2007; Fu et al. 2009; Marcon-Uchida et al. 2010). Some models predict that the radial gradients steepen with time (Chiappini et al. 1997, 2001), while other models predict that the radial gradients flatten with time (Molla et al. 1997; Hou et al. 2000; Prantzos \& Boissier 2000; Fu et al. 2009). The reason for these opposite predictions is that CEM models are very sensitive to the prescriptions of the detailed physical processes that lead to the chemical enrichment of inner and outer disks. Observational constraints on these physical processes are lacking.

Metallicity gradient evolution is unknown due to the small angular sizes and the strong decline of surface brightness of distant galaxies. Before the next generation of telescopes, the only way to overcome this observational hurdle is to combine the magnification power of gravitational lensing with the Laser Guide Star Adaptive Optics (LGSAO) aided integral field spectrograph units (IFUs) on the world's largest telescopes (Jones et al. 2010b; Stark et al. 2008; Swinbank et al. 2007). Recently, Jones et al. (2010a) reported the metallicity gradient of a strongly lensed dispersion-dominated galaxy at high- $z$ ("the Clone arc" at $z=2.00$ ). Jones et al. (2010a) measured the slope of the gradient for the inner $\sim 1 \mathrm{kpc}$ and found it to be considerably steeper than local disk galaxies. The steep gradient at high- $z$ is consistent with the "inside-out" disk formation scenarios. However, in a low resolution study, Cresci et al. (2010) reported "inverted" metallicity gradients for three nonlensed $z \sim 3$ Lyman-break galaxies (LBGs), arguing for a "coldflow" of primordial gas to the galactic core regions. Clearly, a large sample of galaxies with chemical abundance gradients across cosmic time are required to place robust constraints on disk formation theories.

In this Letter, we report the first metallicity gradient measurement of a lensed spiral galaxy at $z=1.49$ based on our OH-Suppressing Infrared Imaging Spectrograph (OSIRIS) observations at KECK II. Throughout this Letter, we use a $\Lambda$ CDM cosmology with $H_{0}=70 \mathrm{~km} \mathrm{~s}^{-1} \mathrm{Mpc}^{-1}, \Omega_{M}=0.30$, and $\Omega_{\Lambda}=0.70$. At $z=1.5,1$ arcsec corresponds to $8.5 \mathrm{kpc}$ and a look-back time of $9.3 \mathrm{Gyr}$. We use solar oxygen abundance $12+$ $\log (\mathrm{O} / \mathrm{H})_{\odot}=8.66$ (Asplund et al. 2005).

\section{OBSERVATIONS AND DATA REDUCTION}

The spiral galaxy is a four-image lensed system first identified by Smith et al. (2009), located behind the massive, X-ray luminous cluster MACS J1149.5+2223. We observed the largest of these four images at $\left(\alpha_{2000}, \delta_{2000}\right)=\left(11^{\mathrm{h}} 49^{\mathrm{m}} 35^{\mathrm{s}} .284\right.$, $+22^{\circ} 23^{\prime} 45^{\prime \prime}$.86) (called Sp1149 hereafter). Smith et al. (2009) construct a detailed mass model for the galaxy cluster and show that $\mathrm{Sp} 1149$ is one of the largest recorded lensed images of a single galaxy at $z>1$. Owing to the lensing magnification and fortuitous face-on orientation, this galaxy exhibits more than 10 locally resolved $\mathrm{H}$ II regions (Figure 1), ideal for spatial 







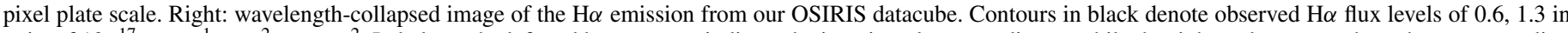

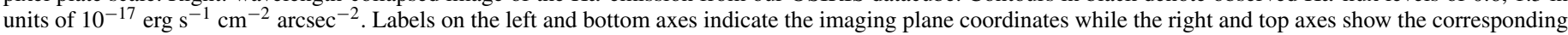


generally well with the rest-frame UV morphology.

(A color version of this figure is available in the online journal.)

chemical abundance studies. The star formation rate (SFR) from the $V_{555}$ band is $\sim 6 M_{\odot} \mathrm{yr}^{-1}$ (Smith et al. 2009).

We observed Sp1149 with the OSIRIS (Larkin et al. 2006) in conjunction with the LGSAO system on KECK II telescope on 2010 March 3. The weather conditions were moderate with the optical FWHM ranging between 0.6 and 1".5. The average tip/tilt corrected FWHM during the observation was $0{ }^{\prime \prime} 1$. To capture $\mathrm{H} \alpha$ and $[\mathrm{N}$ II], we used the narrow-band $\mathrm{Hn} 3$ filter (spectral coverage: $1.594-1.676 \mu \mathrm{m}$; spectral resolution: 2400) and a plate scale of 0.' 1 per lenslet. The observations were conducted in an ABAA dithering sequence with a 10.7 west-north chop onto an object-free sky region. A total of 19 exposures were obtained for the target, with $900 \mathrm{~s}$ of exposure for each individual frame. The net on-target exposure time is therefore $4.75 \mathrm{hr}$. Figure 1 shows the OSIRIS field of view (FOV) and locations of Sp1149 on an HST F814W, F555W color image.

Individual exposures were first reduced using the OSIRIS data reduction pipeline (Larkin et al. 2006). We used the sky subtraction IDL code of Davies (2007), which we have modified to optimize the sky subtraction specifically around the wavelength region of $\mathrm{H} \alpha$ and [N II]. Since we are mainly concerned with the emission lines, a first-order polynomial function was fit to each spatial sample pixel (spaxel) to improve the removal of lenslet to lenslet variations. The final datacube was constructed by aligning the sub-exposures with the centroid of the $\mathrm{H} \alpha$ images and combing using a $3 \sigma$ mean clip to reject cosmic rays and bad lenslets. Telluric correction and flux calibration was performed by observing a few A0V standard stars immediately after the science exposure. The typical uncertainty in flux calibration is $\sim 10 \%$.

Gaussian profiles were fitted simultaneously to the three emission lines: [N II] $\lambda 6548,6583$ and $\mathrm{H} \alpha$. The line profile fitting was conducted using a $\chi^{2}$ minimization procedure which takes into account the greater noise level close to atmospheric $\mathrm{OH}$ emissions. The centroid and velocity width of $[\mathrm{N}$ II $] \lambda \lambda 6548$, 6583 lines were constrained by the velocity width of $\mathrm{H} \alpha \lambda 6563$, and the ratio of $[\mathrm{N}$ II $] \lambda 6548$ and $\left[\mathrm{N}_{\mathrm{II}}\right] \lambda 6583$ is constrained to the theoretical value (Osterbrock 1989). An instrumental profile of $5.9 \AA$ calculated from sky lines has been subtracted from the line widths. We demand a minimum signal-to-noise ratio $(\mathrm{S} / \mathrm{N})$ of 3 to detect each emission line and a minimum $\mathrm{S} / \mathrm{N}$ of 5 for kinematic and metallicity analysis.

\section{KINEMATICS AND LENSING MODELING}

To align the astrometry to the IFU datacube, we first assign the brightest pixel of the $\mathrm{H} \alpha$ narrow-band image with the coordinates of the galactic core from the Hubble Space Telescope (HST) image, and then rotate the IFU datacube with the observing position angle. The $\mathrm{H} \alpha$ image can then be aligned with the HST image by matching coordinates. The upper right panel of Figure 1 shows our observed $\mathrm{H} \alpha$ emission line image, collapsed in wavelength, and smoothed with a Gaussian kernel of $\mathrm{FWHM}=2.5$ pixels. The $\mathrm{H} \alpha$ morphology aligns generally well with the rest-frame NUV/ $U$-band image, especially some of the $\mathrm{H}$ II regions in the west. The SFR and size relation of individual H II regions will be reported in a following paper (R. C. Livermore et al. 2011, in preparation). 
We fit the velocity field with rotation disk models as described in Jones et al. (2010b). The best fit yields a rotation velocity of $V_{\text {rot }} \sin i=150 \pm 15 \mathrm{~km} \mathrm{~s}^{-1}$, with inclination angle $i=$ $45^{\circ} \pm 10^{\circ}$. Using $M_{\mathrm{dyn}}=V_{\mathrm{rot}}^{2} R / G$, the derived dynamical mass is $M_{\text {dyn }}=(2.6 \pm 0.4) \times 10^{10} \quad M_{\odot}$ within $R=2.5 \mathrm{kpc}$. Sp1149 is a rotation-dominated disk with $V_{\text {rot }} / \sigma \sim 4$.

To reconstruct the source-plane morphology and obtain flux magnification for Sp1149, we apply the most recent lens model for cluster MACS J1149.5+2223 as described in Smith et al. (2009). The derived luminosity weighted magnification for Sp1149 is $\mu=22 \pm 2$. Accounting for the lensing amplification, the intrinsic magnitude in the $I$ band is $I \simeq 23.4 \pm 0.3$, corresponding to $M_{B} \simeq-20.7$.

Sp1149 is stretched $\sim 5$ on each side $(x, y)$ of the twodimensional image. Because of this fortuitous uniform magnification, we can work with the observed datacube and divide a factor of 5 linearly and a factor of 22 in flux to recover the intrinsic linear geometry and luminosity of the galaxy. Our results are therefore unchanged when we use the reconstructed source-plane datacube.

\section{METALLICITY GRADIENT}

For the metallicity analysis in this work, we use the empirical strong-line diagnostic-the $\mathrm{N} 2=\log \left(\left[\mathrm{N}_{\mathrm{II}}\right] \lambda 6583 / \mathrm{H} \alpha\right)$ index, as calibrated by Pettini \& Pagel (2004, hereafter PP04):

$$
12+\log (\mathrm{O} / \mathrm{H})=8.90+0.57 \times \mathrm{N} 2 .
$$

We note that the $\mathrm{N} 2$ ratio is sensitive to shock excitation, the ionization state of the gas, and the hard ionization radiation field from an active galactic nucleus (Kewley \& Dopita 2002; Kewley et al. 2006; Rich et al. 2010). Our N2 ratios are consistent with star formation throughout the disk. The ratio of $[\mathrm{N} \mathrm{II}] / \mathrm{H} \alpha$ for the whole galaxy is $0.112 \pm 0.050$.

Due to its relatively low metallicity (compared with local spirals), the [N $\mathrm{NI}]$ line is too weak to reach a $\mathrm{S} / \mathrm{N}$ of 5 in individual OSIRIS pixels, but is clearly detected. We therefore integrate the spectrum within three annuli (see Figure 1, right panel). The physical lengths of the three outer radii of each annulus in the source plane are $r 1=0.72 \pm 0.1 \mathrm{kpc}, r 2=$ $2.34 \pm 0.2 \mathrm{kpc}$, and $r 3=4.5 \pm 0.4 \mathrm{kpc}$. The [N II] line is robustly detected at $\mathrm{S} / \mathrm{N}>5$ for the inner two annuli and is a $3 \sigma$ detection for the outer annulus which we give as an upper limit. The final spectra for the three annuli are presented in Figure 2.

The metallicities converted from the N2 calibrator for the three annuli are

$$
12+\log (\mathrm{O} / \mathrm{H})= \begin{cases}8.54 \pm 0.04 & \text { for } r<r 1 \\ 8.38 \pm 0.05 & \text { for } r 1<r<r 2 \\ <8.05 & \text { for } r 2<r<r 3 \\ & (3 \sigma \text { upper limit })\end{cases}
$$

The "global" metallicity measured from the integrated spectrum of all three regions is $8.36 \pm 0.04$. Note that the errors of line flux measurements are smaller than the intrinsic 0.18 dex scatter pertinent to the $\mathrm{N} 2$ calibration (Erb et al. 2006). The systematic uncertainty of the $\mathrm{N} 2$ calibration with respect to other line diagnostics is about 0.04 dex (Kewley \& Ellison 2008).

A linear regression with censored data yields a metallicity gradient for the central $4.5 \mathrm{kpc}$ of $\frac{\Delta \log (\mathrm{O} / \mathrm{H})}{\Delta R}=-0.16 \pm$ $0.02 \mathrm{dex} \mathrm{kpc}^{-1}$, or $-0.15 \mathrm{dex} \mathrm{kpc}^{-1}$ if we treat the last data point

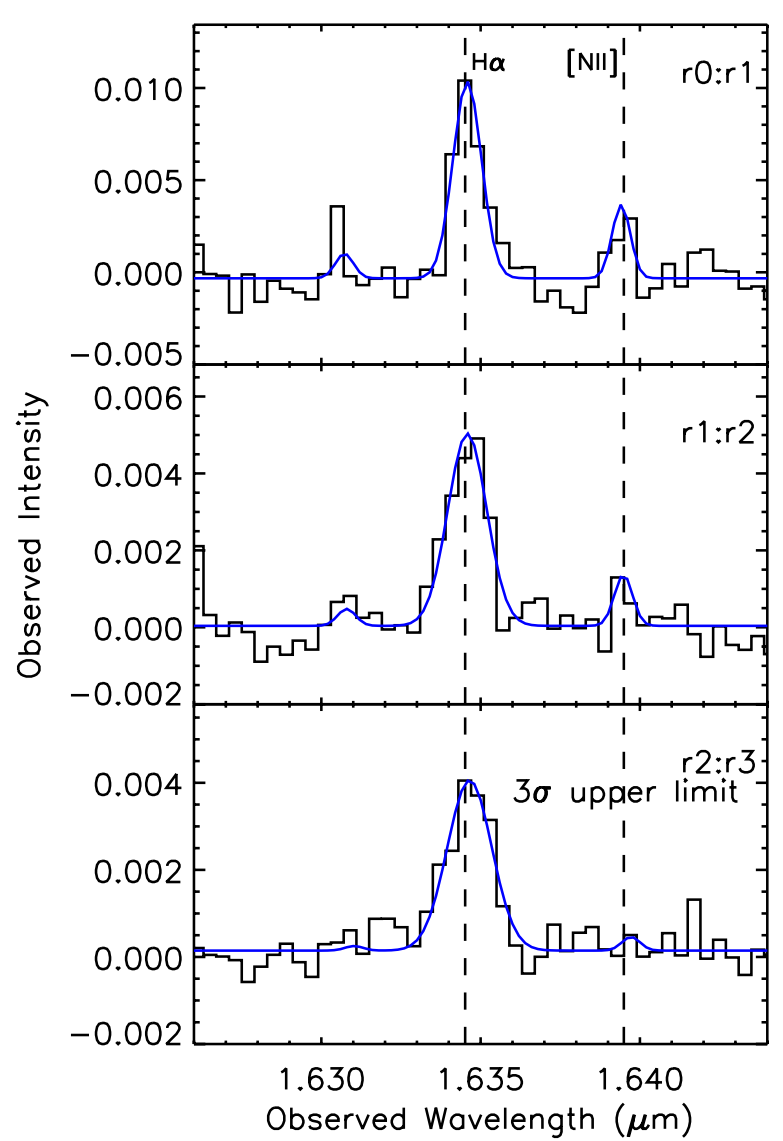

Figure 2. Integrated spectra for the three annuli defined in Figure 1. Vertical dashed lines indicate the zero-velocity positions of [N $\mathrm{II}]$ and $\mathrm{H} \alpha$. The $y$-axis is the observed intensity in units of $10^{-17} \mathrm{erg} \mathrm{s}^{-1} \mathrm{~cm}^{-2} \AA^{-1}$. The annuli radii $r 1$, $r 2$, and $r 3$ are chosen to ensure a $\mathrm{S} / \mathrm{N}$ ([N $\left.\mathrm{NI}_{\mathrm{II}}\right]$ ) to be $>5$ for the inner two radii. Blue curves are the Gaussian fits of the spectra. Note that the outer annulus $r 2: r 3$ is a $3 \sigma$ upper limit for [N $\mathrm{II}]$. The declining ratio of [N $\mathrm{II}] / \mathrm{H} \alpha$ from the central to the outer disk infers a decreasing metallicity with galactocentric distance.

(A color version of this figure is available in the online journal.)

as a measurement rather than an upper limit. The true gradient may be steeper than this value given the $3 \sigma$ upper limit in the outer annulus.

\section{METALLICITY GRADIENT COMPARED WITH LOCAL AND HIGHER REDSHIFT}

In Figure 3 we compare the metallicity gradient of Sp1149 with galaxies in the local and higher redshift universe, as well as the model predictions from Prantzos \& Boissier (2000). To avoid systematic effects caused by different calibration indicators, we use the oxygen abundance of H II regions for comparison. Kewley \& Ellison (2008) showed that metallicities can be compared if the same metallicity calibration is applied consistently across all sample galaxies and $\mathrm{H}$ II regions. We convert all metallicities to the PP04 calibration using the calibrations in Kewley \& Ellison (2008).

Prantzos \& Boissier (2000) modeled metallicity gradients of spiral galaxies at ages $t=3,7.5$, and $13.5 \mathrm{Gyr}$, for different velocities $\left(V_{c}\right)$ and spin parameters $(\lambda)$. Since $S p 1149$ is at an age of $\sim 4.3 \mathrm{Gyr}$ and has $V_{c}=212 \pm 50 \mathrm{~km} \mathrm{~s}^{-1}$, we adopt model grids with $V_{c}=220 \mathrm{~km} \mathrm{~s}^{-1}, t=3,13.5 \mathrm{Gyr}$, and $\lambda=0.03,0.05$, 0.07 for our comparison. The model metallicities have been calibrated to the PP04 metallicity diagnostic. Sp1149 shows a steeper gradient than predicted by Prantzos \& Boissier (2000) 

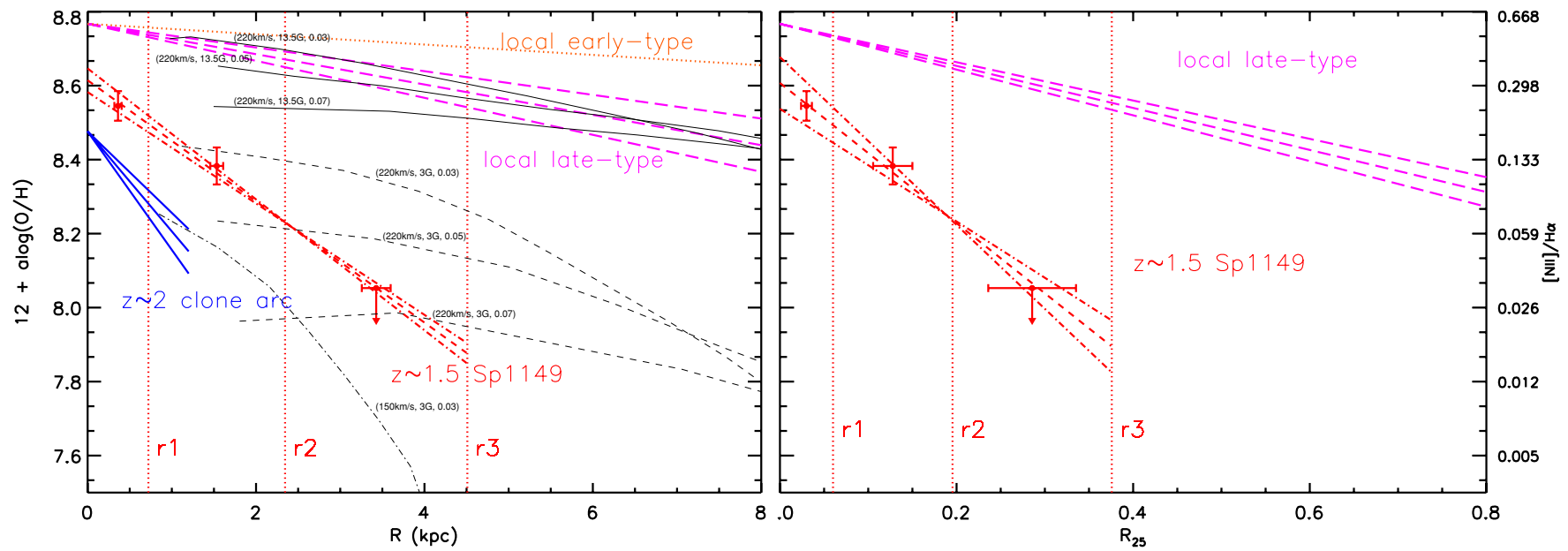

Figure 3. Left: metallicity vs. galactocentric radius. Red lines are the measurements for $\mathrm{Sp} 1149$ at $z=1.49$ from this work. The gradient within the central $4.5 \mathrm{kpc}$ is $-0.16 \pm 0.02 \mathrm{dex} \mathrm{kpc}^{-1}$. Vertical red dotted lines show the annulus used to average/sum the spectra. Purple dashed lines show the typical gradients of local isolated late-type galaxies, using the control sample of Rupke et al. (2010b). The orange dotted line represents the mean gradient of local early-type galaxies, which is typically $\sim 3$ times shallower than local late-type galaxies (Henry \& Worthey 1999). Blue lines show the work of Jones et al. (2010a) for a dispersion-dominated lensed galaxy the "Clone Arc" at $z=2.0$ who report a even steeper gradient within a size of $\sim 1 \mathrm{kpc}$. It is interesting to see that two $z>1$ galaxies have considerably steeper gradients than local galaxies. Black lines are the model predictions from Prantzos \& Boissier (2000) for rotational velocity $V_{c}=220 \mathrm{~km} \mathrm{~s}^{-1}$ at age $t=3 \mathrm{Gyr}$ (dashed curves) and $t=13.5 \mathrm{Gyr}$ (solid curves), with spin parameter $\lambda=0.03,0.05,0.07$. The black dash-dotted line is the model grid with velocity $V_{c}=150 \mathrm{~km} \mathrm{~s}{ }^{-1}$ at $t=3 \mathrm{Gyr}$ and $\lambda=0.03$. The steep gradients of Sp1149 and "Clone Arc" are broadly consistent with the shape of the $V_{c}=150 \mathrm{~km} \mathrm{~s}^{-1}$ model grids. Right: the same as the left panel, except that the $x$-axis is expressed in the scaled radius $R_{25}$.

(A color version of this figure is available in the online journal.)

for $V_{c}=220 \mathrm{~km} \mathrm{~s}^{-1}$. However, the steep gradient of Sp1149 is broadly consistent with the $V_{c}=150 \mathrm{~km} \mathrm{~s}^{-1}$ model (the dashdotted model line in Figure 3).

Metallicity gradients in local galaxies are strongly correlated with Hubble type, bar strength, and merging events, with more flattened gradients for barred galaxies and merging pairs (Kewley et al. 2010; Rupke et al. 2010a). Therefore, it is significant that the metallicity gradient of Sp1149 is substantially steeper than local late-type galaxies (see Figure 3). For the local galaxy gradients, we adopt the isolated spiral control sample of Rupke et al. (2010b) comprised of 11 isolated mid-to-late-type local galaxies with a mix of bar strengths. The median slope for the local control sample is $-0.041 \pm 0.009 \mathrm{dex} \mathrm{kpc}^{-1}$, with a standard deviation of $0.03 \mathrm{dex} \mathrm{kpc}^{-1}$. The median slope of gradients from the Rupke et al. (2010b) sample is consistent with the values of Zaritsky et al. (1994) and van Zee et al. (1998) samples. The Zaritsky et al. (1994) gradient sample composed of 39 local disk galaxies, and the van Zee et al. (1998) sample added another 11 local spirals. Local early-type galaxies have even shallower gradients (Henry \& Worthey 1999).

A similar (and even steeper) gradient has been reported in a recent work of Jones et al. (2010a) for a dispersion-dominated lensed system within only $\sim 1 \mathrm{kpc}$ at $z=2$. Cresci et al. (2010) recently reported reversed metallicity gradients (i.e., the core is less enriched than the outer disk) for three unlensed LBGs at $z \sim 3$. They explained the inverted gradients by inflow of pristine cold gas to the galactic center. Due to their low resolution $(\sim 4-6 \mathrm{kpc})$, the LBG selection, and potential presence of mergers and galactic winds which may contaminate metallicity gradient, it is not clear how the Cresci et al. (2010) results relate to the work presented here.

In the right panel of Figure 3, we show that the metallicity gradient of Sp1149 is still considerably steeper than local galaxies when expressed in units of $\operatorname{dex} /\left(R / R_{25}\right)$, where $R_{25}$ is the $B$-band isophotal radius at a surface brightness of $25 \mathrm{mag}$ $\operatorname{arcsec}^{-2}$. We estimate the $R_{25}$ for $\mathrm{Sp} 1149$ by fitting the isophotal

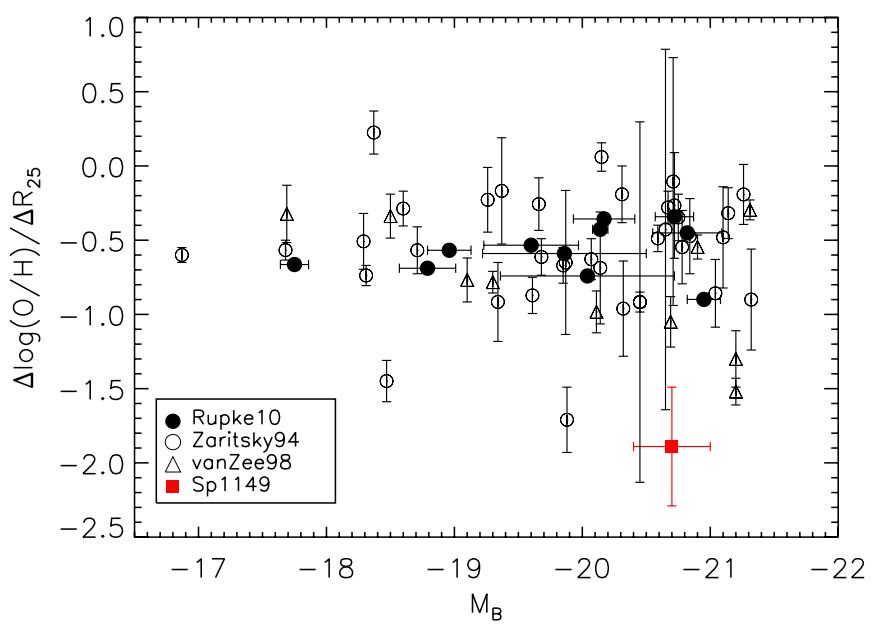

Figure 4. Metallicity gradient $\left(\mathrm{dex} / R_{25}\right)$ vs. absolute $B$-band magnitude $M_{\mathrm{B}}$. The red square represents the measurement for $\operatorname{Sp} 1149$ at $z=1.49$. Black filled circles indicate the locations of the local late-type galaxies of Rupke et al. (2010b). Black empty circles are from the sample of Zaritsky et al. (1994), where the metallicity gradients are measured for 39 local disk galaxies. Black empty triangles come from the sample of van Zee et al. (1998), where the metallicity gradients are provided for another 11 local spiral galaxies. Sp1149 has significantly steeper gradient than local galaxies of comparable luminosity, even when expressed in units of scaled radius.

(A color version of this figure is available in the online journal.)

profile of the source-plane F814W-band image assuming a color difference of $U-B=-0.1 \mathrm{mag}$ for Sc-type galaxies (Fukugita et al. 1995). The resulting $R_{25}$ for $S p 1149$ is $12 \pm 2 \mathrm{kpc}$, comparable to the range of $R_{25}$ in our local control sample (Kewley et al. 2010; Rupke et al. 2010b).

To analyze the effect of size and luminosity on the metallicity gradients, in Figure 4, we plot the metallicity gradients (in units of $R_{25}$ ) versus the absolute $B$-band magnitude $M_{\mathrm{B}}$ for local late-type galaxies and Sp1149. Sp1149 is comparable to the brightest local late-type disks, while "the Clone Arc" is much 
more luminous $\left(M_{\mathrm{B}}=-22.12\right)$. We see from Figure 4 that the scaled gradient of Sp1149 is steeper than in typical local galaxies.

\section{DISCUSSION}

The redshift range of $z=1-3$ is the period when star formation, mass assembly, and chemical enrichment activity peaks (e.g., Fan et al. 2001; Dickinson et al. 2003; Chapman et al. 2005; Hopkins \& Beacom 2006; Conselice et al. 2007). Metallicity gradient studies of galaxies in this redshift range therefore give important insights into disk galaxy formation. The picture that the metallicity gradient flattens with cosmic time is qualitatively consistent with the "inside-out" disk formation scenario (e.g., Prantzos \& Boissier 2000; Bouwens et al. 1997; Benson 2010). This scenario predicts that in the early stages of galaxy evolution, the inner galactic bulge undergoes a rapid collapse with vigorous star formation at the center, building a steep radial metallicity gradient from the core to the outer disk. Violent gas dynamics and accretion events marked these early stages of disk formation. Subsequent radial mixing and infall cause the gradient to flatten over the following Gyr, resulting in a weaker gradient on average in late-type galaxies today.

In this work, we have presented an OSIRIS IFU study of a grand-design lensed spiral galaxy at $z=1.49$. The $22 \times$ magnification allows us to resolve individual $\mathrm{H}$ II regions at an intrinsic resolution of $170 \mathrm{pc}$. We measure the radial distribution of chemical abundances in three annuli and find that the metallicity decreases from a central value of $12+\log (\mathrm{O} / \mathrm{H})=$ 8.54 to less than 8.05 in the outer disk. The metallicity slope is $-0.16 \pm 0.02 \mathrm{dex} \mathrm{kpc}^{-1}$ for the inner $4.5 \mathrm{kpc}$, much steeper than that of late-type galaxies of comparable luminosities in the local universe, even when expressed in $R_{25}$ units. Our results are broadly consistent with the "inside-out" model of disk formation.

Metallicity gradient observations for a larger number of starforming galaxies at different redshifts are required to build a well-defined sample to form a solid observational picture of the metallicity gradient evolution as a function of cosmic time. Before the next generation of telescopes, we are aiming to secure a few tens of gravitationally lensed systems as a pioneer sample to study the gradient evolution of galaxies at high- $z$. Future instruments such as the JWST, TMT, and GMT will improve the current resolution limits by $\sim 1$ order of magnitude, revolutionizing chemical gradient evolution studies at high- $z$.

We thank the referee for his/her valuable comments which have helped a lot to improve this Letter. This work is based on data obtained at the W. M. Keck Observatory. We are grateful to the Keck Observatory staff for assistance with our observations, especially Jim Lyke, Hien Tran, and Randy Campbell. T.-T.Y. thanks the hospitality of ICC, Durham University, where a large portion of the work was done. A.M.S. acknowledges an STFC
Advanced Fellowship. R.C.L. acknowledges a studentship from STFC. The authors recognize the very significant cultural role that the summit of Mauna Kea has within the indigenous Hawaiian community.

\section{REFERENCES}

Asplund, M., Grevesse, N., \& Sauval, A. J. 2005, in ASP Conf. Ser. 336, Cosmic Abundances as Records of Stellar Evolution and Nucleosynthesis, ed. T. G. Barnes, III \& F. N. Bash (San Francisco, CA: ASP), 25

Benson, A. J. 2010, Phys. Rep., 495, 33

Bouwens, R. J., Cayon, L., \& Silk, J. 1997, ApJ, 489, L21

Chapman, S. C., Blain, A. W., Smail, I., \& Ivison, R. J. 2005, ApJ, 622, 772

Chiappini, C., Matteucci, F., \& Gratton, R. 1997, ApJ, 477, 765

Chiappini, C., Matteucci, F., \& Romano, D. 2001, ApJ, 554, 1044

Conselice, C. J., et al. 2007, MNRAS, 381, 962

Cresci, G., Mannucci, F., Maiolino, R., Marconi, A., Gnerucci, A., \& Magrini, L. 2010, Nature, 467, 811

Davies, R. I. 2007, MNRAS, 375, 1099

Dickinson, M., Papovich, C., Ferguson, H. C., \& Budavári, T. 2003, ApJ, 587, 25

Erb, D. K., Shapley, A. E., Pettini, M., Steidel, C. C., Reddy, N. A., \& Adelberger, K. L. 2006, ApJ, 644, 813

Fan, X., et al. 2001, AJ, 121, 54

Fu, J., Hou, J. L., Yin, J., \& Chang, R. X. 2009, ApJ, 696, 668

Fukugita, M., Shimasaku, K., \& Ichikawa, T. 1995, PASP, 107, 945

Henry, R. B. C., \& Worthey, G. 1999, PASP, 111, 919

Hopkins, A. M., \& Beacom, J. F. 2006, ApJ, 651, 142

Hou, J. L., Prantzos, N., \& Boissier, S. 2000, A\&A, 362, 921

Jones, T., Ellis, R., Jullo, E., \& Richard, J. 2010a, ApJ, 725, L176

Jones, T. A., Swinbank, A. M., Ellis, R. S., Richard, J., \& Stark, D. P. 2010b, MNRAS, 404, 1247

Kewley, L. J., \& Dopita, M. A. 2002, ApJS, 142, 35

Kewley, L. J., \& Ellison, S. L. 2008, ApJ, 681, 1183

Kewley, L. J., Groves, B., Kauffmann, G., \& Heckman, T. 2006, MNRAS, 372 , 961

Kewley, L. J., Rupke, D., Jabran Zahid, H., Geller, M. J., \& Barton, E. J. 2010, ApJ, 721, L48

Larkin, J., et al. 2006, New Astron. Rev., 50, 362

Magrini, L., Corbelli, E., \& Galli, D. 2007, A\&A, 470, 843

Marcon-Uchida, M. M., Matteucci, F., \& Costa, R. D. D. 2010, A\&A, 520, 35

Molla, M., Ferrini, F., \& Diaz, A. I. 1996, ApJ, 466, 668

Molla, M., Ferrini, F., \& Diaz, A. I. 1997, ApJ, 475, 519

Osterbrock, D. E. 1989, in Astrophysics of Gaseous Nebulae and Active Galactic Nuclei (Research Supported by the University of California, John Simon Guggenheim Memorial Foundation, University of Minnesota, et al.; Mill Valley, CA: University Science Books), 422

Pettini, M., \& Pagel, B. E. J. 2004, MNRAS, 348, L59

Prantzos, N., \& Boissier, S. 2000, MNRAS, 313, 338

Rich, J. A., Dopita, M. A., Kewley, L. J., \& Rupke, D. S. N. 2010, ApJ, 721, 505

Rupke, D. S. N., Kewley, L. J., \& Barnes, J. E. 2010a, ApJ, 710, L156

Rupke, D. S. N., Kewley, L. J., \& Chien, L. 2010b, ApJ, 723, 1255

Searle, L. 1971, ApJ, 168, 327

Shields, G. A. 1974, ApJ, 193, 335

Smith, G. P., et al. 2009, ApJ, 707, L163

Stark, D. P., Swinbank, A. M., Ellis, R. S., Dye, S., Smail, I. R., \& Richard, J. 2008, Nature, 455, 775

Swinbank, A. M., Bower, R. G., Smith, G. P., Wilman, R. J., Smail, I., Ellis, R. S., Morris, S. L., \& Kneib, J.-P. 2007, MNRAS, 376, 479

van Zee, L., Salzer, J. J., Haynes, M. P., O’Donoghue, A. A., \& Balonek, T. J. 1998, AJ, 116, 2805

Zaritsky, D., Kennicutt, Jr., R. C., \& Huchra, J. P. 1994, ApJ, 420, 87 